\section{Static wavelength scanning using tunable external cavity laser diode}

\author{
Takamasa Suzuki, ${ }^{a}$ MEMBER SPIE, Ryuichi Nagai, ${ }^{a}$ and \\ Osami Sasaki, ${ }^{\text {b }}$ FELLOW SPIE \\ aNiigata University, Graduate School of Science and \\ Technology, 8050 Ikarashi 2, Niigata, 950-2181 Japan \\ ${ }^{\mathrm{b}}$ Niigata University, Engineering, 8050 Ikarashi, 2 Niigata, \\ 950-2181 Japan. \\ E-mail: takamasa@eng.niigata-u.ac.jp
}

Abstract. An external-cavity laser diode that performs static wavelength scanning eliminates problems such as repeatability and tuning rate that arise due to mechanical movements induced in the external cavity of conventional systems, because it requires no mechanical elements. Experiments reveal that the scanning range and tuning rate are $1.1 \mathrm{~nm}$ and $1 \mathrm{kHz}$, respectively. (c) 2010 Society of PhotoOptical Instrumentation Engineers.

[DOI: $10.1117 / 1.3306642]$

Subject terms: wavelength scanning; laser diode; acousto-optic deflector; external cavity.

Paper 090799LR received Oct. 14, 2009; revised manuscript received Jan. 5, 2010; accepted for publication Jan. 8, 2010; published online Feb. 18, 2010.

\section{Introduction}

Wide-range wavelength scanning is one of the key techniques to improve the performance of optical devices such as spectroscopic instruments, optical coherence tomography systems, and interferometers. Generally, wide-range wavelength scanning can be carried out by using a wavelength-selective element such as a diffraction grating and tuning mirror with a gain medium. ${ }^{1}$ Since the cavity of a laser diode (LD) is compact and susceptible to the external cavity, it is favorable for use as a gain medium. ${ }^{2}$ In particular, if an output facet of the LD is processed with an antireflection (AR) coating, the LD is strongly coupled to the external cavity. ${ }^{3-5}$ Tiziani, Franze, and Haible proposed a Littman-cavity-based light source that provided a continuous wavelength tuning range of $25 \mathrm{~nm}$ by using an ARcoated LD. ${ }^{6}$ A tuning range of $4 \mathrm{~nm}$ was obtained with an intracavity glass plate as the fine tuning device, and an AR-coated 633-nm LD in the Littrow configuration. ${ }^{7}$

In particular, since the output facet of the commercially available conventional LD has been processed with an AR coating, the cavity of the LD can be coupled to an external cavity easily as it is. Harvey and Myatt proposed an external-cavity laser diode (ECLD) that consists of a commercial LD in the Littman configuration; the tuning range of this LD was greater than $20 \mathrm{~nm} .{ }^{8}$ We have proposed a simple Littman-type ECLD capable of stabilizing the wavelength through a feedback control ${ }^{9}$ and a Littrow-type ECLD for laser cooling and trapping. ${ }^{10}$ Standard LDs are used in these configurations.

However, we need to focus on the repetition rate, repeatability, tuning speed, and temporal stability of wavelength

0091-3286/2010/\$25.00 @ 2010 SPIE

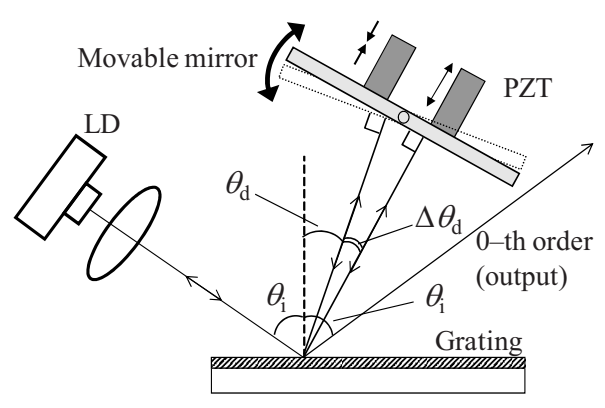

(a)

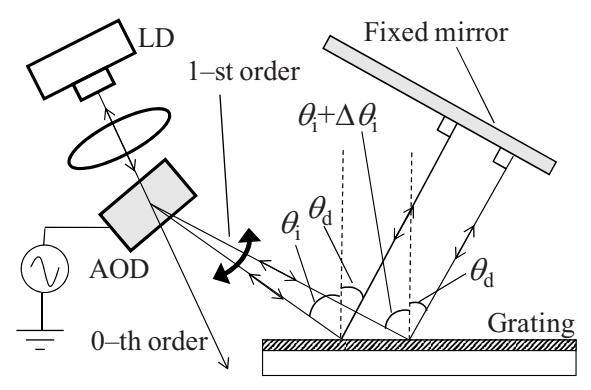

(b)

Fig. 1 Schematic of (a) conventional ECLD and (b) proposed system.

scanning, because ECLDs usually induce mechanical movements that can affect the optical alignment and piezoelectric transducers (PZT) that have hysteresis characteristics. These movements affect the robustness of the cavity and the hysteresis deteriorates the repeatability. The repetition rate and tuning speed were limited by the mechanical movements. To overcome these problems, an electrooptical arrangement was set up in the Littrow configuration. ${ }^{11,12}$ In Ref. 11, an electro-optical crystal was inserted into the cavity instead of mechanically moving the grating. The electric field applied to the crystal changes the refractive index, which in turn tunes the wavelength. The tuning range and speed of this system were $0.01 \mathrm{~nm}$ and $3 \times 10^{-3} \mathrm{~nm} / \mu \mathrm{s}$, respectively, with the special AR-coated LD. Although the system is simple, the tuning range is small. The combination of a liquid crystal cell and a $\mathrm{LiNbO}_{3}$ crystal is used in the external cavity in Ref. 12, in which a tuning range of $10.3 \mathrm{~nm}$ was obtained.

In this work, we propose another type of ECLD that consists of an acousto-optical element instead of an electrooptical crystal. As our system also requires mechanical movements to be restricted, static wavelength scanning can be carried out; thus, the problems arising from the mechanical movements are resolved.

\section{Principle}

Generally, wide-range wavelength scanning can be carried out with the configuration shown in Fig. 1(a), in which a mechanical rotating mirror driven by a PZT is used. The fundamental equation in Fig. 1(a) is represented by ${ }^{13}$ 


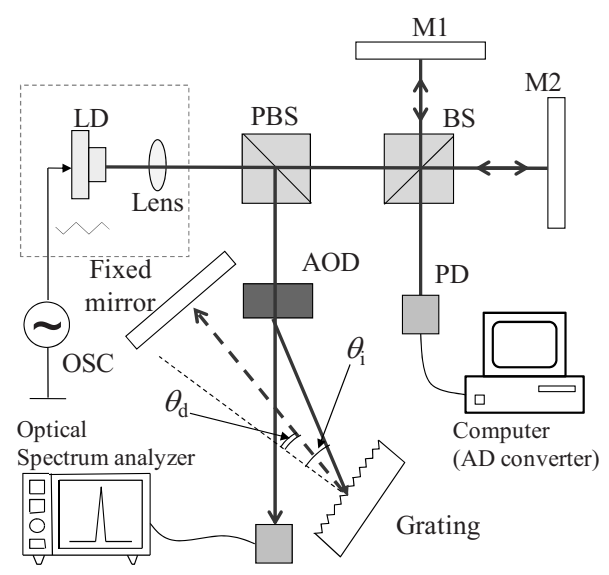

Fig. 2 Experimental setup. LD, laser diode; PBS, polarizing beamsplitter; BS, beamsplitter; OSC, oscillator; PD, photodiode; AOD, acousto-optic deflector.

$\lambda=d\left(\sin \theta_{i}+\sin \theta_{d}\right)$,

where $\lambda$ is the wavelength, $d$ is the groove spacing, and $\theta_{i}$ and $\theta_{d}$ are the incident and diffraction angles, respectively. When the incident angle is constant, the wavelength varies, as given by

$\Delta \lambda=d\left[\sin \left(\theta_{d}+\Delta \theta_{d}\right)-\sin \theta_{d}\right]$,

depending on $\Delta \theta_{d}$, which is the change in the diffraction angle. In this case, the cavity length is nearly unchanged.

Our proposed system is shown in Fig. 1(b). As seen in the figure, wavelength scanning is controlled by the incident angle. The scanning is accomplished statically because an acousto-optic deflector (AOD) is used for the incident angle control. In this case, the wavelength change $\Delta \lambda$ is given by

$\Delta \lambda=d\left[\sin \left(\theta_{i}+\Delta \theta_{i}\right)-\sin \theta_{i}\right]$.

The cavity length varies slightly with the change of incident angle in this setup.

\section{Experiments}

In the setup shown in Fig. 2, we used a commercial LD having an AR-coated facet. The central wavelength and typical threshold current of the LD is $658 \mathrm{~nm}$ and $65 \mathrm{~mA}$, respectively. The output power is proportional to the operating current that is larger than the threshold current. It reaches $80 \mathrm{~mW}$ at $150 \mathrm{~mA}$ of operating current. The changes in the temperature of the LD were restricted to deviations of $\pm 0.01{ }^{\circ} \mathrm{C}$ by means of a Peltier thermocontroller. The output beam from the LD is collimated with a microscope objective lens. The central frequency of the acoustic wave is $75 \mathrm{MHz}$. The deflection angle observed at the AOD varies by $0.18^{\circ} / \mathrm{V}$ with the control voltage. The diffraction efficiency of the AOD is $75 \%$. The groove spacing of the holographic diffraction grating, initial incident angle $\theta_{i}$, and diffraction angle $\theta_{d}$, were $1 / 1800 \mathrm{~mm}$, $63 \mathrm{deg}$, and $18 \mathrm{deg}$, respectively. The diffraction efficiency at the grating is $45 \%$. Since the first-order beam diffracted by the grating is reflected on the stationary mirror and fed back to the LD, the oscillating wavelength is strongly affected by the external cavity. To realize the resonant condi-

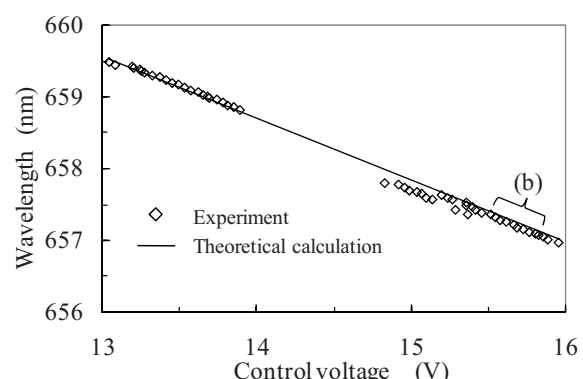

(a)

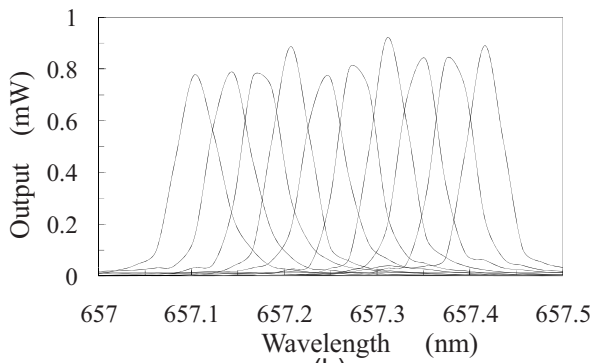

(b)

Fig. 3 Result of wavelength scan. (a) Wavelength shift corresponding to the AOD control voltage and (b) output spectra observed between 15.4 and $15.8 \mathrm{~V}$ in the higher voltage area.

tion, we adjusted the position of the fixed mirror with the activated AOD. The laser beam collimated by the objective lens passes a polarizing beamsplitter (PBS), AOD, grating, and is reflected back from the fixed mirror. We can estimate that $9 \%$ of fraction is fed back to the LD. The length of the external cavity is $340 \mathrm{~mm}$. It changes by $0.27 \mathrm{~mm} / \mathrm{V}$, because the distance between the AOD and the grating is $76 \mathrm{~mm}$. The zero-order beam is detected by an optical spectrum analyzer (OSA) having a wavelength resolution of $0.005 \mathrm{~nm}$. M1, M2, and BS configure an unbalanced Twyman-Green interferometer, whose optical difference is $\sim 1 \mathrm{~mm}$ for observing wavelength tunability.

We observed a wavelength shift by using the OSA. When the control voltage applied to the AOD was varied from 13.0 to $16.2 \mathrm{~V}$, a wavelength shift was observed, as shown in Fig. 3. The solid line in Fig. 3(a) shows the theoretical calculation using Eq. (3). Stable spectra were observed in the region from 659.5 to $658.8 \mathrm{~nm}$ in the lower control-voltage area $(13.0$ to $13.9 \mathrm{~V})$, and in the region from 657.8 to $656.7 \mathrm{~nm}$ in the higher control-voltage area $(14.8$ to $16.2 \mathrm{~V})$. No stable spectra were observed in the middle range of the control voltage, because the power of the deflected beam decreased considerably in this region, and the oscillation mode could not be coupled to the external cavity. However, in the lower and higher voltage areas, tuning ranges of 0.7 and $1.1 \mathrm{~nm}$ were observed. It was determined that the wavelength scanning rate was $0.78 \mathrm{~nm} / \mathrm{V}$. A trace of part of the observed spectra is shown in Fig. 3(b). These were observed between 15.4 and $15.8 \mathrm{~V}$ in the higher voltage area [Fig. 3(b)] that is indicated in Fig. 3(a). The interval of these spectra and the full width at half maximum (FWHM) were $\sim 0.04$ and $\sim 0.06 \mathrm{~nm}$, respectively. The observed FWHM is almost the same as that of the external cavity less standard operation.

In applications such as spectroscopic instruments, opti- 


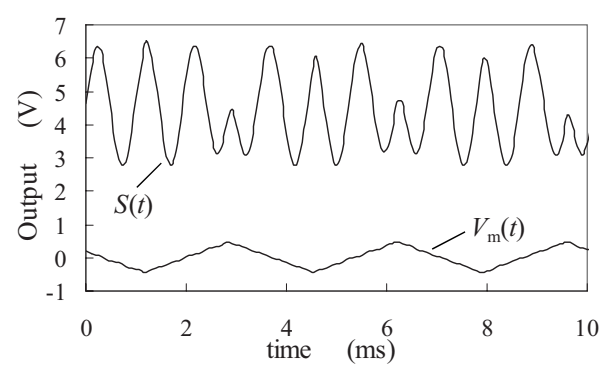

Fig. 4 Observed interference signal $S(t)$ that is modulated with the triangular modulating signal $V_{m}(t)$.

cal coherence tomography, and interferometers, continuous wavelength scanning and tuning speed are important characteristics. We observed an interference signal by using the unbalanced interferometer, and proposed ECLD to confirm these important characteristics. When triangular wavelength scanning was employed as shown in Fig. 4, we observed a continuous interference signal $S(t)$ that exhibits a partially sinusoidal waveform in the linear region of the triangular wave. ${ }^{14}$ The modulation frequency in Fig. 4 was $1 \mathrm{kHz}$, and we were able to observe a stable interference signal up to this frequency. Since the amplitude of the triangular control voltage $\left(V_{m}\right)$ was $0.5 \mathrm{~V}$, the change in the wavelength of the linear part is estimated to be $0.78 \mathrm{~nm}$ from the wavelength-scanning rate, as discussed earlier. This observation of the interference signal confirms that continuous and high-speed wavelength scanning is possible using our system.

\section{Conclusions}

In conclusion, we propose and demonstrate a static type of wavelength-scanning ECLD. Wide-range and continuous wavelength scanning can be carried out under a high tuning rate. In addition, it is found that the use of a special ARcoated LD instead of a standard LD improves the scanning range.

\section{Acknowledgment}

This work was supported by KAKENHI (18560409).

\section{References}

1. M. G. Littman and H. J. Metcalf, "Spectrally narrow pulsed dye laser without beam expander," Appl. Opt. 17, 2224-2227 (1978).

2. M. W. Fleming and A. Mooradian, "Spectral characteristics of external-cavity controlled semiconductor lasers," IEEE J. Quantum Electron. QE-17, 44-59 (1981).

3. R. Wyatt and W. J. Devlin, " $10 \mathrm{kHz}$ linewidth $1.5 \mu \mathrm{m}$ InGaAsP external cavity laser with $55 \mathrm{~nm}$ tuning range," Electron. Lett. 19, 110 112 (1983).

4. X. Gao, Y. Zheng, H. Kan, and K. Shinoda, "Effective suppression of beam divergence for a high-power laser diode bar by an externalcavity technique," Opt. Lett. 29, 361-363 (2004).

5. Y. Zheng and H. Kan, "Effective bandwidth reduction for a highpower laser-diode array by an external-cavity technique," Opt. Lett. 30, 2424-2426 (2005).

6. H. J. Tiziani, B. Franze, and P. Haible, "Wavelength-shift speckle interferometry for absolute profilometry using a mode-hop free external cavity diode laser," J. Mod. Opt. 44, 1485-1496 (1997).

7. Z. Chao, J. Wu, C. Yin, and Y. Xu, "633-nm tunable external-cavity diode laser with an intercavity glass plate as the fine-tuning device," Opt. Eng. 40(5), 801-804 (2001).

8. K. C. Harvey and C. J. Myatt, "External-cavity diode laser using a grazing-incidence diffraction grating," Opt. Lett. 16, 910-912 (1991)

9. T. Suzuki, T. Endo, T. Iwana, and O. Sasaki, "A tunable external cavity laser diode possessing a stable wavelength," Opt. Rev. 14, 23-28 (2007).

10. H. Katori and F. Shimizu, "Laser cooling and trapping of argon and krypton using diode lasers," J. Appl. Phys. 29, L2124-2126 (1990).

11. L. Levin, "Mode-hop-free electro-optically tuned diode laser," Opt. Lett. 27, 237-239 (2002)

12. J. R. Andrews, "Electronically tunable single-mode external-cavity diode laser," Opt. Lett. 16, 732-734 (1991).

13. E. Hecht, "The diffraction grating," in Optics, 3rd ed., pp. 465-476, Addison Wesley Longman, New York (1998).

14. K. Tatsuno and Y. Tsunoda, "Diode laser direct modulation heterodyne interferometer," Appl. Opt. 26, 37-40 (1987). 